\title{
CAN A SINGLE RESOURCE SUPPORT MANY CONSUMER SPECIES ?
}

\author{
SULOCHANA GADGIL AND MADHAV GADGIL \\ Centre for Theoretical Studies, Indian Institute of Science \\ Bangalore 560012
}

\section{$\begin{array}{lllllllllllllllllllllll} & \mathrm{N} & \mathrm{T} & \mathrm{R} & \mathrm{O} & \mathrm{D} & \mathrm{U} & \mathrm{C} & \mathrm{T} & \mathrm{I} & \mathrm{O} & \mathrm{N}\end{array}$}

The number of species which can be packed within a biological community continues to be one of the most intriguing problems in ecology (Volterra 1931, Gause 1934, MacArthur and Levins 1964, Levins 1968, Levin 1970, May and MacArthur 1972, Stewart and Levin 1973). A very general result on this problem states that the number of species in a community cannot exceed the number of limiting factors (Levin 1970). Levin's, as most earlier models are of the form

$$
\frac{1}{x_{i}} \frac{d x_{i}}{d t}=f_{i}\left(z_{1}, \ldots \ldots z_{p}\right)=\sum_{j=1}^{p} a_{i j} z_{j}+\gamma_{i}
$$

Here $x_{i}$ is the density of the $\underline{i}^{\text {th }}$ species and $z_{j}$ is intensity of the $j^{\text {th }}$ limiting factor which in turn is a function of the population densities $x_{1}, \ldots \ldots x_{n}$ and the externally determined resources $y_{1}, \ldots \ldots y_{m}$. The p limiting factors determine the growth rates $f_{1}, \ldots \ldots \ldots f_{n}$ completely. Levin has shown that (i) when the number ' $p$ ' of limiting factors required to determine the growth rates $f_{i}$ is smaller than the total number ' $n$ ' of the species and (ii) when $f_{i}{ }^{\prime} s$ are assumed to be linear functions of the $z_{j}^{\prime} s$; there will be $n-p$ relationships between the population densities $x_{1}, \ldots x_{n}$ which, in general, depend on time and are of the form

$$
x_{1}^{a} 1 x_{2}^{a} x_{3}^{a} \ldots \ldots x_{n}^{a} n=K e^{b t}
$$

When it is assumed, in addition, that (iii) all the populations are non-vanishing and remain bounded for all time, the, value of $b$ in equation (2) has to equal zero and the equation becomes independent of time. In this case, even when eyuilibrium points exist, they will not be asymptotically stable and as such the possibility of a stable co-existence of $\underline{n}$ species is ruleci out when (i), (ii) and (iii) hold.

It is important to note that this result depends crucially on the linearity assumption and will not in general, be valid for nonlinear systems. 
linearities present in realistic models of biological communities limit severely the applicability of this result in understanding the diversity of these communities. Furthermore the assumption of boundedness of $x_{i}{ }^{\prime} s$, which is also crucial to the proof is an additional assumption which may or may not be reasonable for the systems modelled by (1). In fact, we have presented a model in this paper $i n$ which it is not necessary to expect $x_{i}{ }^{\prime} s$ to remain bounded although the governing equation is of the type (1).

The important question that remains is: under what conditions can a single resource support more than one species? From a biological view point this will occur when both of the following conditions are satisfied.

(i) The relative efficiency of two competing species in utilizing a resource depends upon the density of the resource. Thus one species may be more efficient in utilizing a resource at a low density while the other may be more efficient at a high resource density (see e.g., fig. 1).

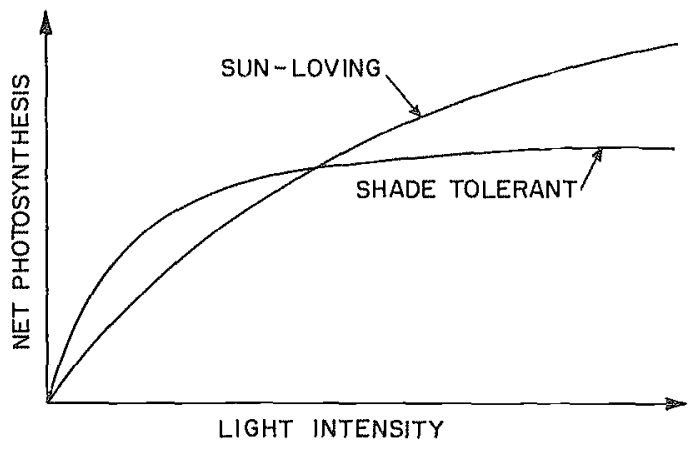

Figure 1. A diagramatic representation of the rate of net photosynthesis as a function of light intensity for a sun-loving versus a shade-tolerant plant.

(ii) There is a persistent temporal or spatial heterogeneity in the density of the resource.

Clearly, for a community in which two species with properties described in (i) are initially present and a temporal or spatial variation in the density of the resource is externally imposed, the final state will contain a mixed population for a certain range of parameters. This is shown by Roughgarden (1971) for a genetic system and by Stewart and Levin (1973) for a community supported by a time-dependent resource. It is important to note that co-existence is rendered possible in such a model only when the spatial or temporal variation of the resource is externally maintained i. e. only in the presence of a favourable boundary condition imposed by forces 
outside the community. Stewart and Levin have shown that for their model the number species cannot exceed the number of resources when the regource inputs are uniform in space and time.

Now if it can be shown that for a resource which is supplied uniformly in space and time, the community itself generates the heterogeneity in resource density which in turn supports the diversity, then we have a mechanism to maintain the diversity which is not crucially dependent on the externally imposed boundary conditions. Such a mechanism will clearly add another dimension to the extent of diversity possible in a community. The most familiar example of a situation in which spatial variation is generated internally within the biological community is the vertical gradient of light intensity in a plant community: In this paper, we develop a mathematical model of the inter- and intra-species interaction within a plant community in the presence of a uniform light intensity at the top of the canopy, and demonstrate the possibility of co-existence of more than one species supported by a single resource for this system.

\section{PLANT COMPETITION}

We use an extension of Cohen's (1971) model of growth and seed production in a plant with a limited growing period as our basic model. Thus, defining,

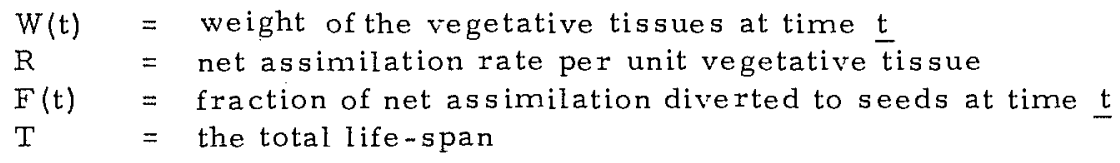

The growth rate and the rate of seed production are given by

$$
\begin{array}{ll}
\frac{\mathrm{dW}}{\mathrm{dt}} & =R(1-F) W \\
\frac{\mathrm{dS}}{\mathrm{dt}} & =R F W
\end{array}
$$

For a discrete model Cohen (1971) has shown that the optimal sequence of allocation of the resources to seed production consists of no allocation upto a transition time $\bar{t}$ and total allocation after $\bar{t}$. Thus, the life history comprises a phase of exclusive vegetative growth followed by a phase of exclusive reproductive growth.

We will assume this for our model also and take

$$
F(t)=0 \quad \frac{t}{t}<\bar{t}<\mathrm{t}
$$


Integration of equations (3) and (4), yields

$$
\begin{aligned}
& W(t)=W(0) e^{R E} \quad t \geqq \bar{t} \\
& S(t)=R W(0) e^{R \bar{t}}(T-\bar{t})
\end{aligned}
$$

The total seed production $\underline{S(T)}$ is maximized when

$$
\frac{\mathrm{d} S(\mathrm{~T})}{\mathrm{dt}}=0
$$

This implies

$$
\begin{aligned}
& \overline{\mathrm{t}}=\mathrm{T}-\mathrm{I} / \mathrm{R} \\
& {[\mathrm{S}(\mathrm{T})] \text { maximum }=W(0) \mathrm{e}^{R(T-1 / R)}}
\end{aligned}
$$

Consider now two plants $\underline{i}$ and $j$ growing side by side and competing for light. We assume that their heights are proportional to the weights of their vegetative tissues $\underline{W}_{i}$ and $\underline{W}_{j}$. The larger plant will be unaffected by the smaller one in this competition but the smaller one will be shaded by the larger plant and its photosynthesis will be depressed. This may be modelled by assuming the growth of the smaller plant $i$ to be given by

$$
\begin{array}{lll}
\mathrm{t}<\overline{\mathrm{t}}_{\mathrm{i}} & \frac{\mathrm{dW_{i }}}{\mathrm{dt}}=\mathrm{R}_{\mathrm{i}} \mathrm{W}_{\mathrm{i}}\left(\mathrm{w}_{\mathrm{i}} / \mathrm{w}_{\mathrm{j}}\right)^{\mathrm{m}} \\
\overline{\mathrm{t}}_{\mathrm{i}<\mathrm{t}<\mathrm{T}} & \frac{\mathrm{dS}}{\mathrm{dt}}=\mathrm{R}_{\mathrm{i}} \mathrm{w}_{\mathrm{i}}\left(\mathrm{w}_{\mathrm{i}} / \mathrm{w}_{j}\right)^{\mathrm{m}}
\end{array}
$$

where $m>0 . \quad$ The growth of the larger plant will be given as before by

$$
\begin{array}{ll}
t<\bar{t}_{j} & \frac{d W_{j}}{d t}=R_{j} W_{j} \\
\bar{t}_{j}<t<T & \frac{d S_{j}}{d t}=R_{j} W_{j}
\end{array}
$$

Note that the index $m$ determines the extent to which the photosynthesis is depressed by the shading. Since this depression will be greater in sunloving plants in comparison with the shade tolerant plants we take $m=I$ for the former and $m<I$ for the latter type of plants. This disadvantage to the sun-loving plants will be counterbalanced by a higher rate of net photosynthesis when exposed to full sunlight. We therefore, assume that the value of $R$ for the sun-loving plants is greater than that for shade-tolerant plants. 
Competition between two sun-loving plants

Consider the competitive interactions of two sun-loving, plants i.e plants with $\underline{m=I}$ when they have germinated simultaneously and have the same biomass $W(0)$ at the initial instant. Let $i$ cease its vegetative growth first, at $t=E_{i}$ and $j$ cease its vegetative growth some time later at $\underline{t}_{j}$ where

$$
\overrightarrow{\mathrm{t}}_{j}=\overline{\mathrm{t}}_{\mathrm{i}}+\mathrm{a} ; \mathrm{a}>\mathrm{o}
$$

The two plants grow side by side upto the instant $\bar{t}_{i}$, neither shading the other till $t=\bar{t}_{i}$. Beyond that, the seed production of $i$ will be affected by the shading by $j$ whereas $j$ will remain unaffected by the presence of $i$ throughout its life history. Hence using (6)

$$
S_{i}(T)=R \int_{\bar{t}_{i}}^{\bar{t}_{j}} \frac{w_{i}^{2}\left(\bar{t}_{i}\right) d t}{w_{j}(t)}+R \int_{\bar{t}_{j}}^{T} \frac{w_{i}^{2}\left(\bar{t}_{i}\right) d t}{w_{j}\left(\bar{t}_{j}\right)}
$$

This is given by

$$
\begin{aligned}
& S_{i}(T)=W(0) e^{R \bar{t}_{i}}\left[1+e^{-a R}\left[\left(T-\bar{t}_{i}-a\right) R-I\right]\right. \\
& S_{j}(T)=R W(0) e^{R\left(\bar{t}_{i}+a\right)}\left(T-\bar{t}_{i}-a\right)
\end{aligned}
$$

Define

$$
D_{i j}\left(\bar{t}_{i}, a\right)=S_{i}(T)-S_{j}(T)
$$

Then

$$
D_{i j}\left(\bar{t}_{i,} a\right)=W(0) e^{R \bar{t}_{i}}\left[1-e^{-a R}-2 R\left(T-\bar{t}_{i}-a\right) \sinh (a R)\right]
$$

The function $D_{i j}$ represents the difference between the total seed-outputs of the plants $-{ }_{i j}$ and $j$ where the $i^{\text {th }}$ plant is assumed to stop its vegetative growth before $i$. It is clear from (8) that $D_{i j}$ is a monotonically. increasing function of $\underline{E}_{i}$, the time at which the $\underline{t}^{t h}$ plant starts seed production. Furthermore,

$$
\begin{aligned}
& D_{i j}\left(\vec{t}_{i}=T-1 / R-a\right)=I-e^{a R}<0 \\
& D_{i j}\left(\vec{t}_{i}=T-a\right)=I-e^{-a R}>0
\end{aligned}
$$

This implies that the seed production of the th plant is less than that of the $j^{\text {th }}$ plant when the $j^{\text {th }}$ starts seed production at $T-1 / R$. This is only to be expected since the seed production of the $\mathrm{j}^{\text {th }}$ plant will be the maximum 
possible in this case. On the other hand, when the $\underline{i}^{\text {th }}$ plant stops its vegetative growth at $T-a$, the $j^{\text {th }}$ plant spends its entire Iife-span $T$ in vegetative growth and hence the seed production of the $i^{\text {th }}$ plant is clearly greater than that of $j$. In between these two extremes is a point $\hat{t}_{i}$ so that when $\bar{t}_{i}=\hat{t}_{i}$ the seed production of the $i^{\text {th }}$ plant equals that of $j^{\text {th }}$ plant. Since we expect both the competitors to have evolutionary flexibility, each of them will choose a life history which will imply a minimum disadvantage in terms of their seed production relative to the competitor. Thus $\frac{i}{S}$ will try to maximise $S_{i}(T)-S_{j}(T)$ and $j$ will try to maximize $\bar{S}_{j}(T)-S_{i}(T)$. The outcome of this competition may then be expected to be

$$
S_{i}(T)=S_{j}(T)
$$

Now (l0) is trivially satisfied when we take

$$
\overline{\mathrm{t}}_{\mathrm{i}}=\overline{\mathrm{t}}_{\mathrm{j}}
$$

The other contour along which (10) holds is given from (8) as

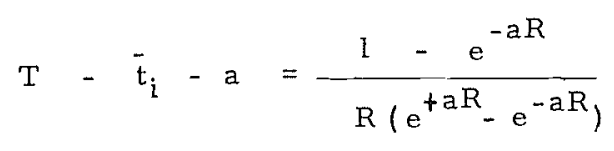

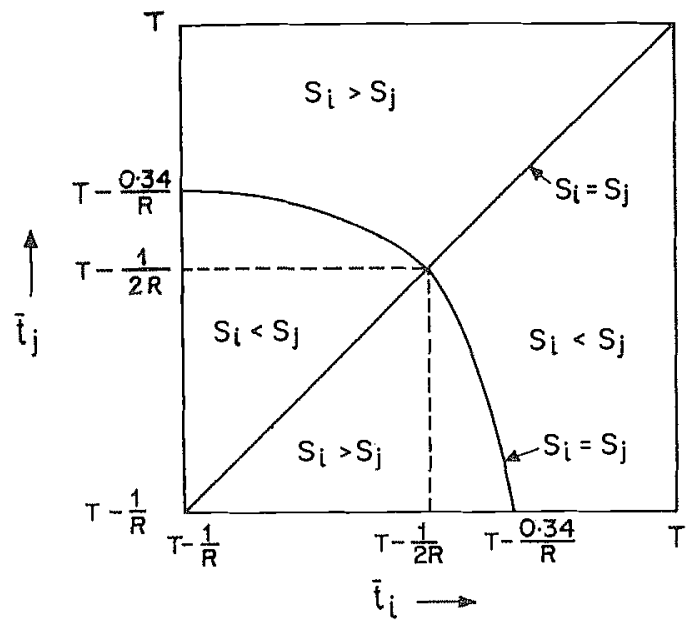

Figure 2. Seed production till the end of growth perlod $S(T)$, for two competitors $i$ and $j$ as a function of the time of transition Ifrom vegetative growth to seed production. 
In Figure 2, both (11) and (12) are drawn in a $\left(\bar{t}_{i}, \bar{t}_{j}\right)$ plane. Note that the intersection of (II) and (I2) occurs at $\overrightarrow{\mathrm{t}}_{\mathrm{i}}$ which can be readily obtained from ( 12 ) by taking the limit as a tends to zero.

$$
\hat{\mathrm{t}}_{\mathrm{i}}=\underset{\mathrm{a} \rightarrow 0}{\mathrm{~L}_{\mathrm{t}}}\left[\mathrm{T}-\frac{\mathrm{I}}{2 \mathrm{R}}-\mathrm{a}+0\left(\mathrm{a}^{2}\right)\right]
$$

Hence

$$
\hat{\mathrm{t}}_{\mathrm{i}}=\mathrm{T}-\mathrm{I} / 2 \mathrm{R}=\hat{\mathrm{t}}_{j}
$$

The point (13) is a saddle point and it can be seen that the strategy of minimizing the disadvantage in seed production will lead to the choice of the value (13) for $\bar{t}_{i}$ and $\bar{t}_{j}$ by the following argument. If the $i$ th plant chooses $\tilde{t}_{i}$ to be $\hat{t}_{i}$ then $S_{i}(\bar{T}) \geqslant S_{i}(T)$ whereas for any other value of $\bar{t}_{i}$, the $j$ th plant can choose a $t_{j}$ which will make its seed output greater than that of $i$ i.e. $S_{i}(T)<S_{j}(T) . \quad T h e$ argument is symmetrically true for $j$ and the plant $j$ will again choose $\underline{t}_{j}$ to be equal to $\hat{t}_{j}$. Thus the evolutionary outcome will be that both the plants will start seed production at the instant given by (13) and will achieve the same seed output and hence a relative fitness equal to one. It is seen that the competition between these plants has delayed the time of transition from vegetative to seed production from $T-I / R$ for the noncompeting plants to $\mathrm{T}-\mathrm{L} / 2 \mathrm{R}$. This implies a decline in the total seed output to $\frac{W(0) e^{R T}-1 / 2}{2}$

Competition in a community of sun-loving and shade-tolerant plants

Consider next the competition between a sun-loving plant, denoted by the subscript $L$ and a shade-tolerant plant, denoted by the subscript $\underline{s}$. Then

$$
\begin{aligned}
& \mathrm{m}_{\mathrm{L}}=1 \\
& \mathrm{R}_{\mathrm{L}}>\mathrm{R}_{\mathrm{s}}
\end{aligned} \quad \mathrm{m}_{\mathrm{g}}<\mathrm{I}
$$

We further assume that the shade-tolerant plants, being less affected by shading are not involved in a race for light as discussed for the sun-loving plants, and stop vegetative growth uniformly at $\mathrm{T}-\mathrm{l} / \mathrm{Rs}$. We also assume $W_{L}(0)=W_{S}(0)$ and the time of germination to be the same. Let $\bar{t}_{i j}$ and Sij (T) be the ${ }_{\text {t }}$ and the seed output of ${ }^{\text {th }}$ plant when competing for light with the $\mathrm{j}^{\text {th }}$ plant, then we have:

$$
\begin{aligned}
& \bar{t}_{s L}=\bar{t}_{s s}=T-I / R s \\
& \bar{t}_{L S}=T-I / R_{L} \\
& \bar{t}_{L I}=T-I / 2 R_{L}
\end{aligned}
$$


Note that (14) implies

$$
\begin{aligned}
& \bar{t}_{L s}>\bar{t}_{s L}
\end{aligned}
$$

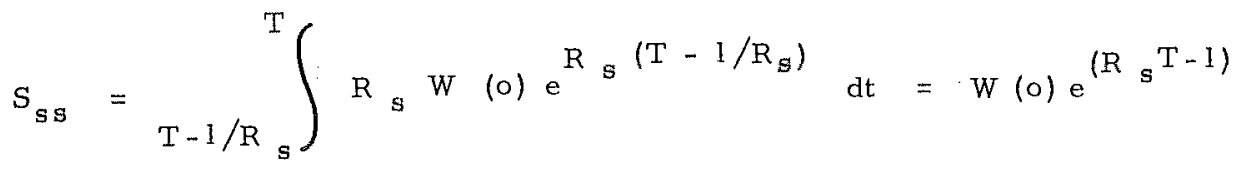

$$
\begin{aligned}
& S_{L L}=\int_{T-1 / 2 R_{L}}^{T} R_{L} W(0) e^{R_{L}\left(T-1 / 2 R_{L}\right)} d t=W(0) e^{\left(R_{L} T-1 / 2\right)}
\end{aligned}
$$

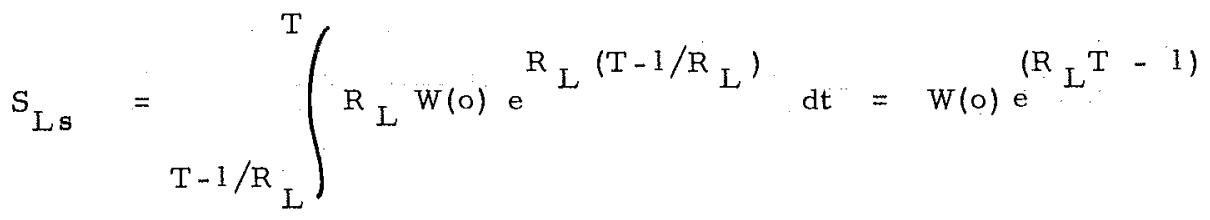

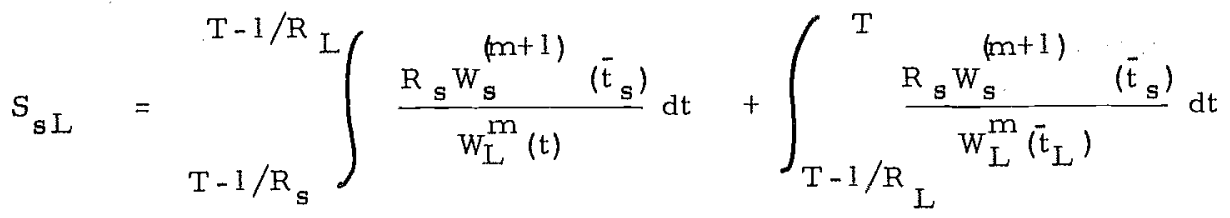

From the above expressions we get by using assumption (14).

$$
\begin{aligned}
& \frac{S_{L s}}{S_{s s}}=e^{\left(R_{L}-R_{s}\right) T}>1 \\
& \frac{s_{s s}}{S_{s L}}=\frac{T-1 / R_{s} R_{s} d t}{\int \frac{T}{T-1 / R_{s}} R_{s}\left[\frac{W_{s}\left(T-1 / R_{s}\right)}{W_{L}(t)}\right]^{m} d t}>1
\end{aligned}
$$

since $W_{B} / W_{L}<1$

Thus

$$
\mathrm{S}_{\mathrm{Ls}}>\mathrm{S}_{\mathrm{ss}}>\mathrm{S}_{\mathrm{sL}}
$$


There is no clear cut inequality between $S_{L L}$ and $S_{s . L}$ Defining

$$
\begin{aligned}
& \mathrm{b}=\mathrm{R}_{\mathrm{s}} / \mathrm{R}_{\mathrm{L}} \\
& \frac{\mathrm{S}_{\mathrm{SL}}}{\mathrm{S}_{\mathrm{LL}}}=\frac{2 \mathrm{be}}{+\mathrm{m}-(\mathrm{m}+1) \mathrm{R}_{\mathrm{L}} \mathrm{T}+1 / 2} \frac{\left(\mathrm{I}-\mathrm{l} / \mathrm{m}\left(1-\mathrm{e}^{-\mathrm{m}(\mathrm{I} / \mathrm{b}-1)}\right)\right.}{\left[\mathrm{I}-\mathrm{b}\left(1-\mathrm{e}^{-\mathrm{mR}} \mathrm{T} \mathrm{T}-\mathrm{m} / \mathrm{b}\right)\right]}
\end{aligned}
$$

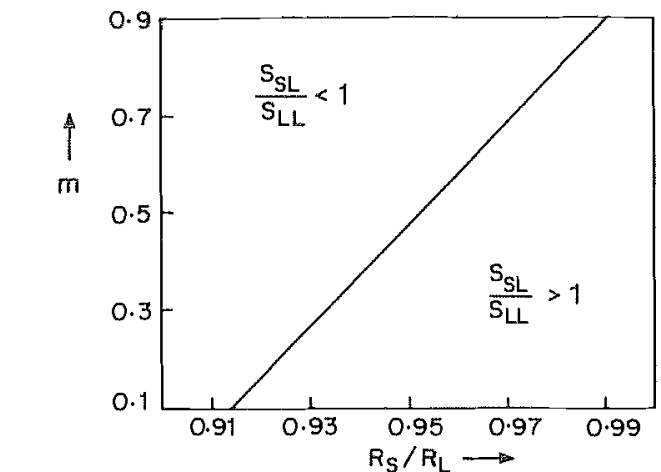

Figure 3. Behaviour of the ratio $S_{S L} / S_{L L}$ as a function of $R_{S} / R_{L}$ and $\mathrm{m}$. $\mathrm{R}_{\mathrm{L}}$ is assumed to be 2.0 , and $\mathrm{T}$ to be 1.0 .

In figure $3, S_{S L} / S_{L L}$ is plotted as a function of $R_{S} / R_{L}$ and $m$. As expected, this ratio increases as the ratio of the netassimilation rates $R_{s} / R_{L}$ increases and decreases as $m$, which measures the detrimental effect of the larger plant on the smaller, increases. It is seen that there is a range of parameter values for which $S_{S L}>S_{L L}$. For this particular range of parameters we have:

$$
S_{L S}>S_{s s}>S_{S I}>S_{L L}
$$

An inspection of this inequality immediately reveals that under these conditions sun-loving and shade-tolerant plants would co-exist because of the frequency dependence of their seed production rates, which may be equated to population growth rates. If a population largely comprises of $L$ plants, then the relevant seed production rates are $S_{S L}$ and $S_{L L}$. Since $S_{S L}>S_{L L}$, the proportion of $s^{t} s$ will increase in such a population. On the other hand, if a population were to be largely made up of $\underline{s}$ plants, the relevant seed production rates are $S_{S s}$ and $S_{L_{S}}$. Since $S_{L_{S}}>S_{S s}$, L's would increase in proportion in such a populatton. In either case then the minority type will tend to increase. We would then expect a co-existence of the two competing types in such a system. 
This co-existence may be shown formally in a model of a mixed population of $I$ and $S$ plants. We assume $I$ and $\underline{s}$ to differ in seed production, but be equivalent in mortality rates. Consider a mixed population of such plants to be held at some constant density through density dependent mortality which does not distinguish between $\underline{s}$ and $\underline{L}$ plants. We may study such a situation by using a model without explicitly introducing the density induced mortality. We then have

$$
\frac{\mathrm{dN}_{\mathrm{S}}}{\mathrm{dt}}=\mathrm{r}_{\mathrm{s}} \mathrm{N}_{\mathrm{S}} ; \quad \frac{\mathrm{dN}_{\mathrm{L}}}{\mathrm{dt}}=\mathrm{r}_{\mathrm{L}} \mathrm{N}_{\mathrm{L}}
$$

$r_{s}$ and $r_{L}$ would be functions of $S_{s s}, S_{s L}, S_{L L}, S_{L s}$ and $N_{s}, N_{L}$.

$$
p_{s}=\frac{N_{s}}{N_{S}+N_{L}}, \quad p_{L}=\frac{N_{L}}{N_{S}+N_{L}}
$$

We do not expect the total population to increase exponentially as implied by (16). But since we assume that the total population is limited by density dependent mortality which does not distinguish between the two categories, and we are mainly interested in the possibility of co-existence, it is sufficient to consider (16) and investigate under what conditions the frequencies $P_{B}$ and $P_{L}$ remain between zero and one. Since we assume that the population density held at a constant level, the incidence of neighbour types would be proportional to their frequency. In that case:

$$
\begin{aligned}
& r_{s}=s_{s s} p_{s}+s_{s L} p_{L} \\
& r_{L}=s_{L s} p_{s}+s_{L L} p_{L}
\end{aligned}
$$

Our complete model then is:

$$
\begin{aligned}
& \frac{d N_{s}}{d t}=N_{s}\left[s_{s s} p_{s}+s_{s L} p_{L}\right] \\
& \frac{d N_{L}}{d t}=N_{L}\left[s_{L s} p_{s}+s_{L L} p_{L}\right]
\end{aligned}
$$

Hence

$$
\frac{d p_{s}}{d t}=\frac{1}{N_{s}+N_{L}} \frac{d N_{s}}{d t}-\frac{N_{s}}{\left(N_{s}+N_{L}\right)^{2}} \frac{d\left(N_{s}+N_{L}\right)}{d t}
$$


i. e.

$$
\begin{aligned}
& \frac{d p_{s}}{d t}=p_{s}\left(1-p_{s}\right)\left[S_{s L}-S_{L L}+\left(S_{s s}+S_{L L}-S_{s L}-S_{L s}\right) p_{s}\right] \\
& \frac{d p_{L}}{d t}=-\frac{d p_{s}}{d t}
\end{aligned}
$$

In this formalism, equilibrium implies a steady frequency and both types of plants will co-exist provided that there exists a steady non-zero frequency less than one for each of them. Note that from (18), we get

$$
\frac{\mathrm{dp}}{\mathrm{dt}}=0 \text { at } \mathrm{p}_{\mathrm{s}}=0,1, \hat{\mathrm{p}}_{\mathrm{s}}
$$

where

$$
\hat{\mathrm{P}}_{s}=\frac{\mathrm{S}_{L L}{ }^{-S} S_{S L}}{\mathrm{~S}_{L L}-S_{s L}+S_{S s}-S_{I s}}
$$

Both species will co-exist if

$$
0<\hat{\mathrm{p}}_{\mathrm{s}}<1
$$

Co-existence is possible under two sets of conditions

$$
\begin{aligned}
& \mathrm{s}_{\mathrm{ss}}>\mathrm{S}_{\mathrm{Ls}} \text { and } \quad \mathrm{s}_{\mathrm{LL}}>\mathrm{s}_{\mathrm{sL}} \\
& \text { or } \mathrm{s}_{\mathrm{SS}}<\mathrm{s}_{\mathrm{LS}} \text { and } \mathrm{s}_{\mathrm{LL}}<\mathrm{s}_{\mathrm{SL}}
\end{aligned}
$$

\section{Stability}

We have shown above that there are three points of equilibrium; two corresponding to populations consisting of one type alone and the last one $\mathrm{p}_{\mathrm{s}}$ corresponding to a mixed population. We investigate now the stability of all these points in order to determine the conditions under which a mixed population will result from an arbitrary initial frequency.

Define

$$
\begin{aligned}
& M=\hat{S}_{s s}+S_{L L}-S_{S L}-S_{L s} \\
& p_{s}=\hat{p}_{s}+t
\end{aligned}
$$


From (18) and (20)

$$
\frac{d p_{s}}{d t}=M p_{s}\left(1-p_{s}\right)\left(p_{s}-\hat{p}_{s}\right)
$$

Equation (24) gives

$$
\frac{\mathrm{d} t}{\mathrm{dt}}=\mathrm{Mp}_{\mathrm{s}}\left(1-\mathrm{p}_{\mathrm{s}}\right) t+0\left(t^{2}\right)
$$

Using (21), we get

$$
\frac{1}{f} \frac{\mathrm{d} f}{\mathrm{dt}}<0 \quad \text { if } M>0
$$

Thus the perturbation $t$ will grow if $\underline{M}>0$ and decay if $\underline{M}<0$ implying that the equilibrium will be stable for the latter case. Note that this implies that the equilibirum is stable when (23) holds and unstable when (22) holds. Similarly it can be shown that the points $\underline{p_{s}}=0,1$ are stable when $\underline{M}>0$ and unstable when $\underline{M}<0$.

To sum up:

(1) $S_{S s}>S_{L s}$ and $S_{L L}>S_{L_{s}}$ implies that the points $\hat{\mathrm{P}}_{s}=0$ and

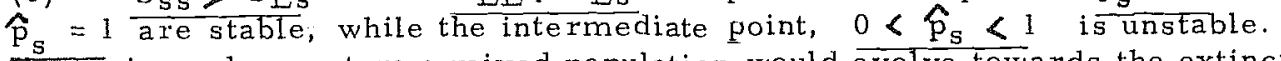
Hence in such a system a mixed population would evolve towards the extinction of one or the other species depending on the initial conditions (fig $4 a$ ).

(a)
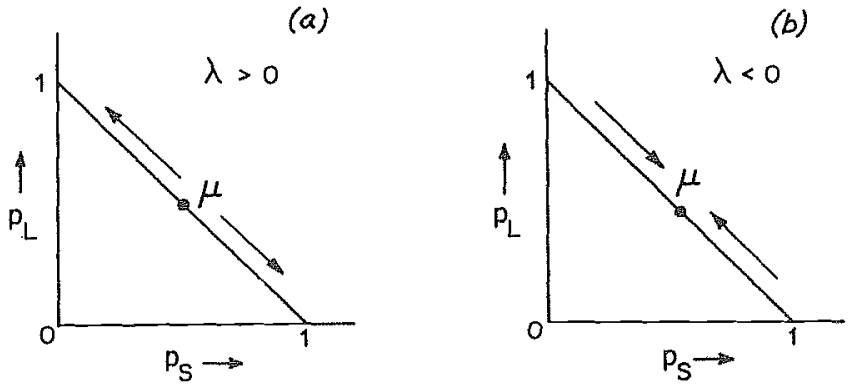

Figure 4. Phase diagram to illustrate the possible equilibria for the aystem of equations (18) and (19). The three posaible points of equilibria at which $d p_{\mathrm{g}} / d t=d p_{L} / d t=0$ are are $\underline{p}_{s}=1, p_{L}=1$ or some $\underline{p}_{B}=\mu$ where $0<M r l$. (a) when $M>0$, the first two are atable and $\bar{M}$ is unstable and (b) when $M<0$ the first two are unstable and $\mu$ is stable. 
(2) $S_{S S}<S_{L_{S}}$ and $S_{L L}<S_{S L}$ implies that the points $\hat{p}_{S}=0$ or $\hat{\mathrm{p}}_{\mathrm{s}}=1$ are points of unstable equilibria, while the intermediate equilibrium point $0<\hat{\mathrm{p}}_{\mathrm{S}}<1$ is stable. Such a system would permit the permanent coexistence of the two competing species (fig 4 b).

It may be mentioned that for this situation the population of species $L$ is related to that of species $\underline{s}$ through a relation similar to (2) viz

$$
\begin{aligned}
& \frac{N_{s}}{N_{I}^{A}} \quad\left(\frac{N_{s}}{N_{L}}\right) e_{t=0}^{+B t} ; \\
& A=\frac{S_{S s}-S_{S L}}{S_{L s}-S_{L L}} \quad ; \quad B=\frac{S_{L s} S_{S L}-S_{S s} S_{L L}}{S_{L s}-S_{L L}}
\end{aligned}
$$

However, here nonzero values of $B$ are permitted because the condition of boundedness of individual populations at large time is not imposed. The imposition of an additional density dependent mortality which does not discern between the two competitors will keep the populations of these two species bounded but will not alter the frequency of the final state and hence will also lead to a mixed population for this case.

Our model for the competitive interactions between sun-loving and shade-tolerant plants generated the inequality (15) for certain values of $\underline{R^{\prime} s}$ and $\underline{m}$;

$$
S_{L s}>S_{S s}>S_{S L}>S_{L L}
$$

This is obviously compatible with the set of inequalities (23), and such a system would permit the permanent comexistence of two species limited by a single resource, which is being continually supplied at a constant Ievel. This co-existence is rendered possible by the spatial heterogeneity in the density of that resource generated by the action of the plant community itself.

Such internally induced spatial heterogeneties are particularly likely to occur in the case of sessile organisms such as plants, but need not be restricted to them. For example, teritorial animals may crop their food plants more intensely near the centre of the territory, and less intensely towards periphery. Other animals using these as shelter may then diversify, a species needing denser cover chosing the periphery and a species requiring thinner cover occurring towards the centre of the territory.

\section{SPECIES PACKING}

The problem of packing of species at different levels of resource densities is analogous to. that of packing of species along a resource continum 
analysed by May and MacArthur (1972). These authors consider the problem of packing of species specialised, for example, to take prey of different sizes. The ability of the species to utilize prey of various sizes may be represented by a utilization function along the prey-size axis (fig 5a). They assume that these utilization functions are Gaussian, and have identical areas unde $r$ the curve. They show that unde $x$ these conditions the species may be packed in a variable environment with the peaks of utilization functions separated by a distance roughly equalling one standard deviation. For our

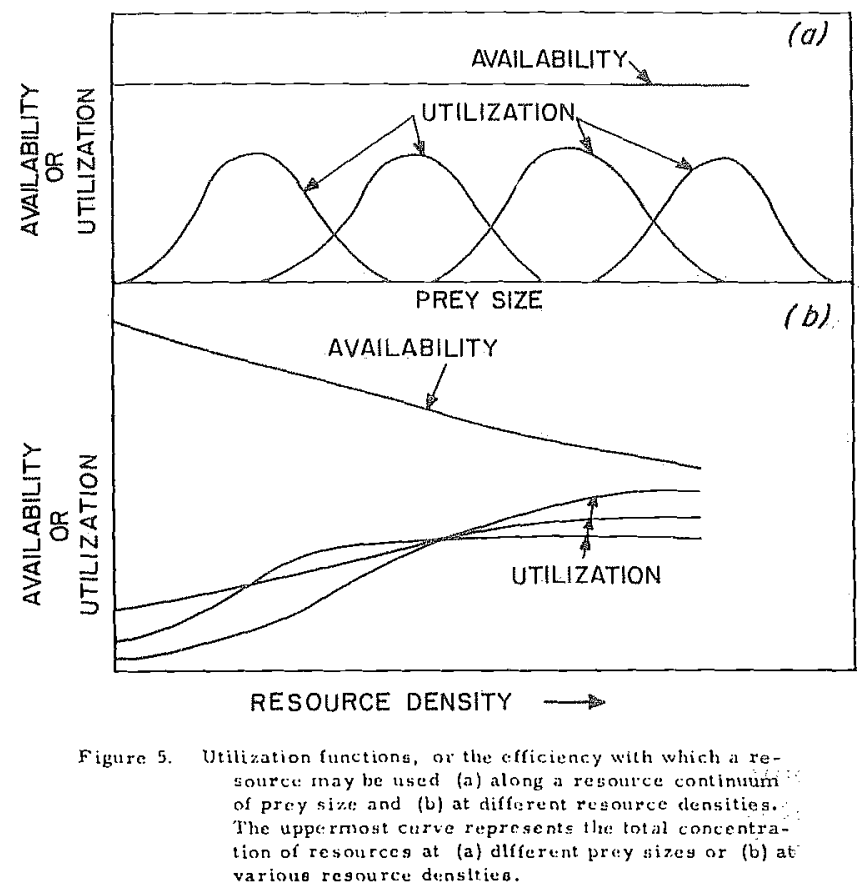

model, we may consider analogous utilization functions along a resource density axis. These could not be Gaussian but would increase monotonically to an asymptote as in the case of the photosynthetic response to light intensity (fig l). In this case, the resource density at which the species is more efficient than all of its competing species would correspond to the peak of the utilization functions of the resource continuum case (fig $5 \mathrm{a}$ and $\mathrm{b}$ ). The problem of how closely the species could be packed in this case deserves to be investigated.

\section{$\underline{\mathrm{U}} \underline{\mathrm{U}} \underline{\mathrm{M}} \underline{\mathrm{M}}$ A}

The conclusion that the number of species co-existing within a biological community cannot exceed the number of limiting factors is not valid if we assume that (i) the relative efficiency of two competing species in utilizing a resource is not inclependent of the resource density, but one species 
may be more efficient at a lower density and less efficient at a higher density and (ii) there is a spatial or temporal heterogeneity in the density of the resource. This spatial or temporal heterogeneity does not have to be furmished by factors external to the biological community, but may be generated within the biological community itself as in the case of a vertical gradient of light in a plant community. This possibility of a stable co-existence of more than one species in a community limited by a single resource, even when the resource is being supplied uniformly in space and time, is formally demonstrated.

$$
\begin{array}{lllllllllll}
R & E & E & E & R & E & N & C & E & S
\end{array}
$$

I COHEN, D. (1971). Maximizing final yield when growth is limited by time or by limiting resources. Journal of Theoretical Biology, 33: 299-307.

2 GAUSE, GF. (1934). The struggle for existence. Williams \& Wilkins, Baltimore, 163 p.

3 LEVIN, S A. (1970). Community equilibria and stability, and an extension of the competitive exclusion principle. Amer Nat, 104: $413-423$.

LEVINS, R. (1968). Evolution in changing environments. Princeton University Press, Princeton, N J. I30p.

5 MACARTHUR, R $\mathrm{H}$ and LEVINS, R. (1964). Competition, habitat selection, and charaçter displacement in a patchy environment. Proc Nat Acad Sci, U S, 5I: 1207-12Lo.

MAY, $R$ and MACARTHUR, $R H$. (1972). Niche overlap as a function of environmental variability. Proc Nat Acad Sci, U S, 69: 1109-I113.

7 ROUGHGARDEN, J. (1971). Density dependent natural selection. Ecology, 52: 453-468.

8 STEWART, $\mathrm{F} N$ and LEVIN, B R. (1973). Partitioning of resources and the outcome of interspecific competition: a model and some general considerations. Amex Nat, 107: $171-198$.

9 VOLTERRA, V. (1931). Lecons sur la theorie mathematique de la Iutte pour la vie. Gauthier - Villars. Paris, 2l 4p. 